\title{
On the dopability of semiconductors and governing materials properties
}

\author{
Anuj Goyal,$^{\dagger}$ Prashun Gorai, ${ }^{\dagger}$ Shashwat Anand,${ }^{\ddagger}$ Eric S. Toberer,${ }^{\dagger}$ G. Jeffrey \\ Snyder, $\stackrel{\ddagger}{\ddagger}$ and Vladan Stevanović ${ }^{*, \dagger}$ \\ $\dagger$ Colorado School of Mines, Golden, CO 80401, USA \\ $\ddagger$ Northwestern University, Evanston, IL, USA \\ E-mail: vstevano@mines.edu
}

\section{Supplementary Information}

\section{Cation vacancy}

The $n$-type dopability metric, Eq. (9) in the main text, based only on cation vacancy is given as:

$$
\delta \varepsilon_{F}^{\left(V_{c}\right)}=\bar{\varepsilon}_{c}-C B M+\frac{1}{N_{c}}\left(\bar{V}_{c}-V_{\alpha}\right)+\frac{1}{N_{c}} \Delta \mu_{c}
$$

Upgrading the model to account for the loss of $N_{c}^{s}$ amount of electrons from the cation- $s$ states $\left(\bar{\varepsilon}_{c}^{s}\right)$ in the valence band of the compound to the reference phase, for systems like $\mathrm{PbX}$ and $\mathrm{Bi}_{2} \mathrm{X}_{3}(\mathrm{X}=\mathrm{S}, \mathrm{Se}, \mathrm{Te})$. Also, assuming cation-rich conditions for the $n$-type doping $\left(\Delta \mu_{c}=0\right)$, changes Eq.(1) to: 


$$
\begin{aligned}
& \delta \varepsilon_{F}^{\left(V_{c}\right)}=\bar{\varepsilon}_{c}-C B M+\frac{N_{c}^{s}}{N_{c}}\left(\bar{\varepsilon}_{c}-\bar{\varepsilon}_{c}^{s}\right)+\frac{1}{N_{c}}\left(\bar{V}_{c}-V_{\alpha}\right), \\
& \delta \varepsilon_{F}^{\left(V_{c}\right)}=\left(1+\frac{N_{c}^{s}}{N_{c}}\right) \bar{\varepsilon}_{c}-C B M-\frac{N_{c}^{s}}{N_{c}} \bar{\varepsilon}_{c}^{s}+\frac{1}{N_{c}}\left(\bar{V}_{c}-V_{\alpha}\right),
\end{aligned}
$$

Using intrinsic material properties as proxies to replace the physically derived terms in Eq.(2). We approach by (1) fitting parameter $\times$ work function of the cation $\left(W_{c}\right), a \times W_{c}$, as a substitute for the $\left(1+\frac{N_{c}^{s}}{N_{c}}\right) \bar{\varepsilon}_{c}$ term (representing average electronic energy of the cation reference phase), (2) $b \times C B M,(3) c \times\left\langle\varepsilon_{c}^{s}\right\rangle$ to substitute $\frac{N_{c}^{s}}{N_{c}} \varepsilon_{c}^{s}$, where $\left\langle\varepsilon_{c}^{s}\right\rangle$ is calculated using computed DOS, (4) and using $d \times \Delta H_{f}$ as representative of the nuclear repulsive term $\frac{1}{N_{c}}\left(\bar{V}_{c}-V_{\alpha}\right)$, and adding an additional fitting parameter $e$ to account for the unknown within the proxies. Eq.(2) now modifies to:

$$
\delta \varepsilon_{F}^{\left(V_{c}\right)}=a^{\left(V_{c}\right)} \times W_{c}-b^{\left(V_{c}\right)} \times C B M-c^{\left(V_{c}\right)} \times \bar{\varepsilon}_{c}^{s}+d^{\left(V_{c}\right)} \times \Delta H_{f}+e^{\left(V_{c}\right)},
$$

where $\left\{a^{\left(V_{c}\right)}, b^{\left(V_{c}\right)}, c^{\left(V_{c}\right)}, d^{\left(V_{c}\right)}, e^{\left(V_{c}\right)}\right\}$ are the free parameters of the model (fitting constants) for the $n$-type dopability metric, when cation vacancy is the lowest energy (killer) intrinsic acceptor defect.

\section{Anion vacancy}

Deriving a more generic description for the anion vacancy formation energy, than that derived in the main text Eq. (6), based on the compound stoichiometry $\mathrm{C}_{x} \mathrm{~A}_{y}$ such that $x N_{c}+y N_{a}=$ $y n_{a}$, where $N=$ electrons and $n=$ states.

$$
\Delta E_{V_{a}}=\frac{x}{y} N_{c}\left(\varepsilon_{F}-\bar{\varepsilon}_{V B}\right)+N_{a}\left(\bar{\varepsilon}_{a}-\bar{\varepsilon}_{V B}\right)+\left(\bar{V}_{a}-V_{\alpha}\right)+\Delta \mu_{a},
$$


p-type dopability metric based on the anion vacancy is then given as:

$$
\delta \varepsilon_{F}^{\left(V_{a}\right)}=\frac{N_{a}}{\frac{x}{y} N_{c}} \bar{\varepsilon}_{a}+V B M-\left(1+\frac{N_{a}}{\frac{x}{y} N_{c}}\right) \bar{\varepsilon}_{V B}+\frac{1}{\frac{x}{y} N_{c}}\left(\bar{V}_{a}-V_{\alpha}\right)+\frac{1}{\frac{x}{y} N_{c}} \Delta \mu_{a}
$$

Assuming anion-rich conditions for $p$-type doping $\left(\Delta \mu_{a}=0\right)$, changes Eq.(5) to:

$$
\delta \varepsilon_{F}^{\left(V_{a}\right)}=\frac{N_{a}}{\frac{x}{y} N_{c}} \bar{\varepsilon}_{a}+V B M-\left(1+\frac{N_{a}}{\frac{x}{y} N_{c}}\right) \bar{\varepsilon}_{V B}+\frac{1}{\frac{x}{y} N_{c}}\left(\bar{V}_{a}-V_{\alpha}\right),
$$

Similar to the $n$-type dopability metric, using proxies in the $p$-type dopability metric. (1) $a \times W_{a}$ (work function of anion reference phase or in case of molecules the first ionization energy) for $\bar{\varepsilon}_{a},(2) b \times V B M,(3)$ average energy (center of mass) of the valence band $c \times \bar{\varepsilon}_{V B}$, and (4) $d \times \Delta H_{f}$ for the nuclear repulsive term $\left(\bar{V}_{a}-V_{\alpha}\right)$. Eq.(6) now modifies to:

$$
\delta \varepsilon_{F}^{\left(V_{a}\right)}=a^{\left(V_{a}\right)} \times W_{a}+b^{\left(V_{a}\right)} \times V B M-c^{\left(V_{a}\right)} \times\left\langle\varepsilon_{a}^{p}\right\rangle+d^{\left(V_{a}\right)} \times \Delta H_{f}+e^{\left(V_{a}\right)},
$$

where $\left\{a^{\left(V_{a}\right)}, b^{\left(V_{a}\right)}, c^{\left(V_{a}\right)}, d^{\left(V_{a}\right)}, e^{\left(V_{a}\right)}\right\}$ are the fitting constants for the $p$-type dopability metric, when anion vacancy is the lowest energy (killer) intrinsic donor defect.

\section{Cation Interstitial}

$$
\begin{gathered}
\Delta E_{I_{c}}=N_{c}\left(\varepsilon_{F}-\bar{\varepsilon}_{c}\right)+N_{c}^{s}\left(\bar{\varepsilon}_{c}^{s}-\bar{\varepsilon}_{c}\right)+\left(V_{\alpha}-\bar{V}_{c}\right)-\Delta \mu_{c} \\
\delta \varepsilon_{F}^{\left(I_{c}\right)}=\varepsilon_{F}-\bar{\varepsilon}_{c}+\frac{N_{c}^{s}}{N_{c}}\left(\bar{\varepsilon}_{c}^{s}-\bar{\varepsilon}_{c}\right)+\frac{1}{N_{c}}\left(V_{\alpha}-\bar{V}_{c}\right)-\frac{1}{N_{c}} \Delta \mu_{c}
\end{gathered}
$$

Ignoring the $\Delta \mu_{c}$ term at the moment, and applying the proxies to rest of the terms in eq. 9. Note that the cation atom likes to donate electrons and hence act like a donor in an ionic material, and will limit the $p$-type dopability. Therefore, assuming Fermi energy to be located at the $V B M$. 


$$
\delta \varepsilon_{F}^{\left(I_{c}\right)}=-a^{\left(I_{c}\right)} \times W_{c}+b^{\left(I_{c}\right)} \times V B M+c^{\left(I_{c}\right)} \times \bar{\varepsilon}_{c}^{s}+d^{\left(I_{c}\right)} \times \Delta H_{f}+e^{\left(I_{c}\right)},
$$

where $\left\{a^{\left(I_{c}\right)}, b^{\left(I_{c}\right)}, c^{\left(I_{c}\right)}, d^{\left(I_{c}\right)}, e^{\left(I_{c}\right)}\right\}$ are the fitting constants for the $p$-type dopability metric, when cation interstitial is the lowest energy (killer) intrinsic donor defect.

\section{Anion Interstitial}

$$
\begin{gathered}
\Delta E_{I_{a}}=N_{a}\left(\bar{\varepsilon}_{V B}-\bar{\varepsilon}_{a}\right)+\frac{x}{y} N_{c}\left(\bar{\varepsilon}_{V B}-\varepsilon_{F}\right)+\left(V_{\alpha}-\bar{V}_{a}\right)-\Delta \mu_{a} \\
\delta \varepsilon_{F}^{\left(I_{a}\right)}=-\frac{N_{a}}{\frac{x}{y} N_{c}} \bar{\varepsilon}_{a}-\varepsilon_{F}+\left(1+\frac{N_{a}}{\frac{x}{y} N_{c}}\right) \bar{\varepsilon}_{V B}+\frac{1}{\frac{x}{y} N_{c}}\left(V_{\alpha}-\bar{V}_{a}\right)-\frac{1}{\frac{x}{y} N_{c}} \Delta \mu_{a}
\end{gathered}
$$

Note that the anion atom likes to accept electrons and hence act like an acceptor, and will limit the $n$-type dopability. Therefore, assuming Fermi energy to be located at the $C B M$ and applying proxies to rest of the terms in eq. 12:

$$
\delta \varepsilon_{F}^{\left(I_{a}\right)}=-a^{\left(I_{a}\right)} \times W_{a}-b^{\left(I_{a}\right)} \times C B M+c^{\left(I_{a}\right)} \times \bar{\varepsilon}_{V B}+d^{\left(I_{a}\right)} \times \Delta H_{f}+e^{\left(I_{a}\right)},
$$

where $\left\{a^{\left(I_{a}\right)}, b^{\left(I_{a}\right)}, c^{\left(I_{a}\right)}, d^{\left(I_{a}\right)}, e^{\left(I_{a}\right)}\right\}$ are the fitting constants for the $n$-type dopability metric, when anion interstitial is the lowest energy (killer) intrinsic acceptor defect. 


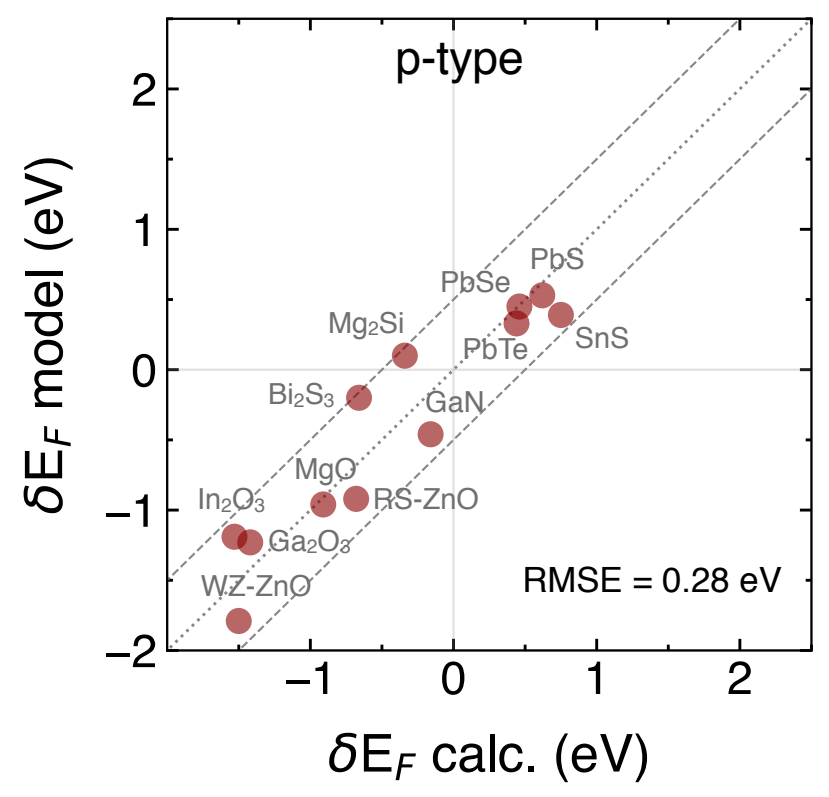

Figure S1: Comparison of the analytic model and the calculated (from first-principles defect calculations) $p$-type dopability metric $\delta \varepsilon_{F}^{(p)}$ in case of cation interstitials. The model parameters for Eq.(10) are obtained via linear regression to the calculated values. The expression for the $p$-type dopability metric based on cation interstitials is given as: $\delta \varepsilon_{F}^{(p)}=$ $-1.41 W_{c}+0.55 V B M+0.01 \bar{\varepsilon}_{c}^{s}-0.09 \Delta H_{f}-3.14$.

Table S1: Experimental data of the band gap and maximal reported charge carrier concentrations for various binary and ternary semiconductors.

\begin{tabular}{|c|c|c|c|c|}
\hline \multirow[t]{2}{*}{ Compound } & \multirow[t]{2}{*}{ Band gap $(\mathrm{eV})$} & \multicolumn{2}{|c|}{ Carrier concentration $\left(\mathrm{cm}^{-3}\right)$} & \multirow[t]{2}{*}{ References } \\
\hline & & $n$ & $p$ & \\
\hline KAlSb4 & 0.20 & $1.00 \mathrm{E}+19$ & - & 1 \\
\hline $\mathrm{AgFeSe}_{2}$ & 0.23 & $5.00 \mathrm{E}+19$ & - & 2 \\
\hline HfNiSn & 0.45 & $1.00 \mathrm{E}+19$ & - & 3 \\
\hline $\mathrm{ZrNiSn}$ & 0.55 & $8.00 \mathrm{E}+19$ & - & 3 \\
\hline TiNiSn & 0.60 & $1.00 \mathrm{E}+19$ & - & 3 \\
\hline $\mathrm{InN}$ & 0.70 & $1.00 \mathrm{E}+19$ & - & 4 \\
\hline $\mathrm{CdO}$ & 0.90 & $1.00 \mathrm{E}+20$ & - & $5-7$ \\
\hline $\mathrm{ZnAs}_{2}$ & 0.90 & $8.00 \mathrm{E}+16$ & - & 8 \\
\hline $\mathrm{ZnSnN}_{2}$ & 0.94 & $1.00 \mathrm{E}+21$ & - & 2,9 \\
\hline $\mathrm{AgInSe}_{2}$ & 0.96 & $2.50 \mathrm{E}+18$ & - & 2 \\
\hline $\mathrm{CdAs}_{2}$ & 1.00 & $7.00 \mathrm{E}+16$ & - & 10 \\
\hline $\mathrm{CdSnP}_{2}$ & 1.16 & $8.00 \mathrm{E}+17$ & - & 2 \\
\hline
\end{tabular}




\begin{tabular}{|c|c|c|c|c|}
\hline $\mathrm{AgInS}_{2}$ & 1.18 & $3.00 \mathrm{E}+20$ & - & 2 \\
\hline $\mathrm{HfCoSb}$ & 1.31 & $1.00 \mathrm{E}+18$ & - & 1 \\
\hline TiPtSn & 1.31 & $1.00 \mathrm{E}+21$ & - & 3 \\
\hline $\mathrm{TiCoSb}$ & 1.42 & $1.00 \mathrm{E}+18$ & - & 1 \\
\hline $\mathrm{Bi}_{2} \mathrm{~S}_{3}$ & 1.45 & $2.00 \mathrm{E}+19$ & - & 11,12 \\
\hline $\mathrm{ZrCoSb}$ & 1.49 & $5.00 \mathrm{E}+19$ & - & 1 \\
\hline $\mathrm{CdIn}_{2} \mathrm{Te}_{4}$ & 1.50 & $3.50 \mathrm{E}+15$ & - & 2 \\
\hline $\mathrm{CdIn}_{2} \mathrm{Se}_{4}$ & 1.55 & $1.50 \mathrm{E}+19$ & - & 2 \\
\hline CdSe & 1.85 & $1.00 \mathrm{E}+18$ & - & 13,14 \\
\hline $\mathrm{Sb}_{2} \mathrm{~S}_{3}$ & 1.88 & $1.00 \mathrm{E}+18$ & - & 15 \\
\hline $\mathrm{ZnIn}_{2} \mathrm{Se}_{4}$ & 1.92 & $8.00 \mathrm{E}+16$ & - & 2 \\
\hline $\mathrm{AgInO}_{2}$ & 2.00 & $3.30 \mathrm{E}+20$ & - & 16,17 \\
\hline $\mathrm{Cd}_{2} \mathrm{SnO}_{4}$ & 2.06 & $1.00 \mathrm{E}+19$ & - & 18,19 \\
\hline $\mathrm{CdIn}_{2} \mathrm{~S}_{4}$ & 2.10 & $1.25 \mathrm{E}+19$ & - & 2 \\
\hline $\mathrm{CdIn}_{2} \mathrm{O}_{4}$ & 2.23 & $1.00 \mathrm{E}+19$ & - & 20 \\
\hline $\mathrm{SiC}-3 \mathrm{C}$ & 2.39 & $1.00 \mathrm{E}+19$ & - & 21,22 \\
\hline $\mathrm{AlP}$ & 2.50 & $1.00 \mathrm{E}+19$ & - & 23 \\
\hline $\mathrm{CdS}$ & 2.56 & $1.00 \mathrm{E}+21$ & - & 2,24 \\
\hline $\mathrm{In}_{2} \mathrm{O}_{3}$ & 2.90 & $3.00 \mathrm{E}+20$ & - & 25,26 \\
\hline $\mathrm{ZnGeN}_{2}$ & 2.90 & $1.00 \mathrm{E}+19$ & - & 9 \\
\hline $\mathrm{TiO}_{2}$ & 3.03 & $1.00 \mathrm{E}+20$ & - & 27,28 \\
\hline $\mathrm{BaSnO}_{3}$ & 3.10 & $1.00 \mathrm{E}+19$ & - & 29 \\
\hline $\mathrm{SrTiO}_{3}$ & 3.25 & $1.00 \mathrm{E}+17$ & - & 30,31 \\
\hline $\mathrm{Zn}_{2} \mathrm{SnO}_{4}$ & 3.35 & $1.00 \mathrm{E}+19$ & - & 32 \\
\hline $\mathrm{MgIn}_{2} \mathrm{O}_{4}$ & 3.40 & $1.00 \mathrm{E}+19$ & - & 33,34 \\
\hline $\mathrm{ZnO}$ & 3.43 & $1.10 \mathrm{E}+21$ & - & 35 \\
\hline $\mathrm{SnO}_{2}$ & 3.60 & $1.00 \mathrm{E}+20$ & - & 36,37 \\
\hline $\mathrm{ZnS}$ & 3.84 & $1.00 \mathrm{E}+18$ & - & 14 \\
\hline $\mathrm{SrSnO}_{3}$ & 3.93 & $1.00 \mathrm{E}+19$ & - & 38,39 \\
\hline $\mathrm{Ga}_{2} \mathrm{O}_{3}$ & 4.90 & $1.00 \mathrm{E}+20$ & - & 40,41 \\
\hline $\mathrm{Bi}_{2} \mathrm{Te}_{3}$ & 0.13 & $1.00 \mathrm{E}+18$ & $1.00 \mathrm{E}+19$ & 42 \\
\hline $\mathrm{Bi}_{2} \mathrm{Se}_{3}$ & 0.16 & $1.00 \mathrm{E}+19$ & $1.00 \mathrm{E}+19$ & $43-45$ \\
\hline $\mathrm{InSb}$ & 0.17 & $1.00 \mathrm{E}+19$ & $1.00 \mathrm{E}+20$ & 46 \\
\hline $\mathrm{CoSb}_{3}$ & 0.22 & $1.00 \mathrm{E}+21$ & $1.00 \mathrm{E}+19$ & 1 \\
\hline $\mathrm{PbSe}$ & 0.27 & $2.00 \mathrm{E}+19$ & $1.00 \mathrm{E}+19$ & 47 \\
\hline
\end{tabular}




\begin{tabular}{|c|c|c|c|c|}
\hline InAs & 0.30 & $1.00 \mathrm{E}+21$ & $1.00 \mathrm{E}+20$ & 46 \\
\hline $\mathrm{CdSnAs}_{2}$ & 0.30 & $5.00 \mathrm{E}+18$ & $5.00 \mathrm{E}+18$ & 2 \\
\hline $\mathrm{PbTe}$ & 0.31 & $1.00 \mathrm{E}+20$ & $1.00 \mathrm{E}+19$ & 48,49 \\
\hline $\mathrm{KGaSb}_{4}$ & 0.39 & $2.50 \mathrm{E}+19$ & $5.00 \mathrm{E}+18$ & 1 \\
\hline $\mathrm{PbS}$ & 0.40 & $4.00 \mathrm{E}+19$ & $8.00 \mathrm{E}+18$ & 50 \\
\hline $\mathrm{Mg}_{3} \mathrm{Sb}_{2}$ & 0.42 & $1.00 \mathrm{E}+19$ & $1.00 \mathrm{E}+20$ & $51-53$ \\
\hline $\mathrm{CoSb}_{3}$ & 0.50 & $1.00 \mathrm{E}+20$ & $1.00 \mathrm{E}+18$ & 1 \\
\hline $\mathrm{CuFeS}_{2}$ & 0.53 & $3.00 \mathrm{E}+21$ & $7.00 \mathrm{E}+20$ & 2,54 \\
\hline $\mathrm{CdGeAs}_{2}$ & 0.53 & $4.00 \mathrm{E}+18$ & $2.00 \mathrm{E}+17$ & 2 \\
\hline $\mathrm{Cu}_{2} \mathrm{SnSe}_{3}$ & 0.66 & $1.00 \mathrm{E}+18$ & $2.90 \mathrm{E}+21$ & 2 \\
\hline $\mathrm{SnO}$ & 0.70 & $1.00 \mathrm{E}+17$ & $1.00 \mathrm{E}+19$ & $55 ?$ \\
\hline $\mathrm{GaSb}$ & 0.73 & $1.00 \mathrm{E}+17$ & $1.00 \mathrm{E}+18$ & $46,56,57$ \\
\hline $\mathrm{Mg}_{2} \mathrm{Si}$ & 0.80 & $1.00 \mathrm{E}+20$ & $5.00 \mathrm{E}+17$ & 58,59 \\
\hline $\mathrm{CuInSe} e_{2}$ & 0.86 & $7.00 \mathrm{E}+18$ & $1.00 \mathrm{E}+19$ & 2 \\
\hline $\mathrm{CuInTe} \mathrm{T}_{2}$ & 0.88 & $2.00 \mathrm{E}+17$ & $7.50 \mathrm{E}+19$ & 2 \\
\hline $\mathrm{SnSe}$ & 0.90 & $2.00 \mathrm{E}+19$ & $2.00 \mathrm{E}+18$ & 60,61 \\
\hline $\mathrm{ScN}$ & 0.90 & $1.00 \mathrm{E}+21$ & $1.00 \mathrm{E}+20$ & $62-64$ \\
\hline $\mathrm{Cu}_{3} \mathrm{~N}$ & 1.00 & $1.00 \mathrm{E}+17$ & $1.00 \mathrm{E}+16$ & 65 \\
\hline $\mathrm{SnS}$ & 1.10 & $4.00 \mathrm{E}+15$ & $1.00 \mathrm{E}+18$ & 66,67 \\
\hline $\mathrm{CuInS}_{2}$ & 1.20 & $1.00 \mathrm{E}+18$ & $2.00 \mathrm{E}+19$ & 2 \\
\hline $\mathrm{InP}$ & 1.34 & $1.00 \mathrm{E}+17$ & $1.00 \mathrm{E}+19$ & 46,68 \\
\hline GaAs & 1.42 & $1.00 \mathrm{E}+18$ & $5.00 \mathrm{E}+19$ & 69,70 \\
\hline $\mathrm{CuInO}_{2}$ & 1.44 & $1.00 \mathrm{E}+17$ & $1.00 \mathrm{E}+17$ & 17,71 \\
\hline $\mathrm{CdTe}$ & 1.58 & $2.00 \mathrm{E}+18$ & $1.00 \mathrm{E}+16$ & $72-75$ \\
\hline $\mathrm{MgGeAs}_{2}$ & 1.60 & $3.00 \mathrm{E}+18$ & $2.00 \mathrm{E}+19$ & 2 \\
\hline $\mathrm{CdGeP}_{2}$ & 1.80 & $1.00 \mathrm{E}+20$ & $2.00 \mathrm{E}+18$ & 2 \\
\hline $\mathrm{ZnSiP}_{2}$ & 2.07 & $4.80 \mathrm{E}+18$ & $5.00 \mathrm{E}+17$ & 2 \\
\hline AlAs & 2.10 & $1.00 \mathrm{E}+17$ & $1.00 \mathrm{E}+19$ & 76 \\
\hline $\mathrm{GaP}$ & 2.30 & $1.00 \mathrm{E}+19$ & $1.00 \mathrm{E}+18$ & 77 \\
\hline $\mathrm{ZnSe}$ & 2.80 & $1.00 \mathrm{E}+19$ & $1.00 \mathrm{E}+17$ & 78,79 \\
\hline $\mathrm{GaN}$ & 3.47 & $5.00 \mathrm{E}+20$ & $5.00 \mathrm{E}+17$ & 80 \\
\hline $\mathrm{Ag}_{2} \mathrm{SnTe}_{3}$ & 0.08 & - & $1.00 \mathrm{E}+20$ & 2 \\
\hline $\mathrm{CuFeTe}_{2}$ & 0.10 & - & $5.00 \mathrm{E}+21$ & 2 \\
\hline $\mathrm{CuFeSe}_{2}$ & 0.16 & - & $2.00 \mathrm{E}+20$ & 2 \\
\hline $\mathrm{SnTe}$ & 0.18 & - & $1.00 \mathrm{E}+21$ & 81 \\
\hline
\end{tabular}




\begin{tabular}{|c|c|c|c|c|}
\hline $\mathrm{Ag}_{2} \mathrm{GeTe}_{3}$ & 0.25 & - & $8.00 \mathrm{E}+17$ & 2 \\
\hline $\mathrm{Sb}_{2} \mathrm{Te}_{3}$ & 0.30 & - & $1.00 \mathrm{E}+19$ & 82 \\
\hline $\mathrm{Cd}_{4} \mathrm{Sb}_{3}$ & 0.30 & - & $3.00 \mathrm{E}+19$ & 1,83 \\
\hline $\mathrm{Cu}_{2} \mathrm{SnSe}_{3}$ & 0.31 & - & $3.20 \mathrm{E}+20$ & 2 \\
\hline $\mathrm{ZnSnSb}_{2}$ & 0.40 & - & $1.00 \mathrm{E}+21$ & 2 \\
\hline $\mathrm{Cu}_{2} \mathrm{SnSe}_{4}$ & 0.50 & - & $1.00 \mathrm{E}+21$ & 2 \\
\hline $\mathrm{Cu}_{2} \mathrm{SnTe}_{3}$ & 0.50 & - & $1.00 \mathrm{E}+21$ & 2 \\
\hline $\mathrm{CdSb}$ & 0.50 & - & $1.00 \mathrm{E}+18$ & 84 \\
\hline $\mathrm{ZnSb}$ & 0.50 & - & $8.00 \mathrm{E}+18$ & 85 \\
\hline $\mathrm{AgAlTe}_{2}$ & 0.56 & - & $1.00 \mathrm{E}+17$ & 2,54 \\
\hline $\mathrm{Cu}_{3} \mathrm{SbS}_{4}$ & 0.74 & - & $1.00 \mathrm{E}+21$ & 2,54 \\
\hline $\mathrm{ZnSnAs}_{2}$ & 0.74 & - & $1.00 \mathrm{E}+21$ & 2,9 \\
\hline $\mathrm{Ag}_{2} \mathrm{SnSe}_{3}$ & 0.81 & - & $1.00 \mathrm{E}+18$ & 2 \\
\hline $\mathrm{Cu}_{2} \mathrm{GeTe}_{3}$ & 0.81 & - & $3.00 \mathrm{E}+21$ & 2 \\
\hline $\mathrm{CuGaTe}_{2}$ & 0.82 & - & $1.10 \mathrm{E}+20$ & 2 \\
\hline $\mathrm{Cu}_{3} \mathrm{AsSe}_{4}$ & 0.88 & - & $2.70 \mathrm{E}+18$ & 2 \\
\hline $\mathrm{Cu}_{2} \mathrm{SnS}_{3}$ & 0.91 & - & $2.50 \mathrm{E}+21$ & 2 \\
\hline $\mathrm{Ag}_{2} \mathrm{GeSe}_{3}$ & 0.91 & - & $2.00 \mathrm{E}+17$ & 2 \\
\hline $\mathrm{Cu}_{2} \mathrm{GeSe}_{3}$ & 0.94 & - & $1.40 \mathrm{E}+21$ & 2 \\
\hline $\mathrm{CuGaSe}_{2}$ & 0.96 & - & $1.20 \mathrm{E}+19$ & 2 \\
\hline $\mathrm{NiO}$ & 1.00 & - & $1.00 \mathrm{E}+19$ & $86-88$ \\
\hline $\mathrm{AgGaSe}_{2}$ & 1.10 & - & $6.60 \mathrm{E}+16$ & 2,54 \\
\hline $\mathrm{Cu}_{2} \mathrm{In}_{4} \mathrm{Te}_{7}$ & 1.10 & - & $5.30 \mathrm{E}+18$ & 2 \\
\hline $\mathrm{ZnGeAs}_{2}$ & 1.16 & - & $1.00 \mathrm{E}+15$ & 9 \\
\hline $\mathrm{Sb}_{2} \mathrm{Se}_{3}$ & 1.20 & - & $1.00 \mathrm{E}+15$ & 89,90 \\
\hline $\mathrm{AgGaTe}_{2}$ & 1.32 & - & $1.00 \mathrm{E}+17$ & 2,54 \\
\hline $\mathrm{Cu}_{3} \mathrm{PSe}_{4}$ & 1.40 & - & $6.00 \mathrm{E}+17$ & 2 \\
\hline $\mathrm{ZnSnP}_{2}$ & 1.45 & - & $1.00 \mathrm{E}+17$ & 2,9 \\
\hline $\mathrm{Cu}_{2} \mathrm{GeS}_{3}$ & 1.50 & - & $3.00 \mathrm{E}+17$ & 2 \\
\hline $\mathrm{CdSiAs}_{2}$ & 1.51 & - & $1.00 \mathrm{E}+17$ & 9 \\
\hline $\mathrm{AlSb}$ & 1.70 & - & $1.00 \mathrm{E}+19$ & 2 \\
\hline $\mathrm{ZnGeP}_{2}$ & 1.80 & - & $1.00 \mathrm{E}+17$ & 9,91 \\
\hline $\mathrm{ZnSiAs}_{2}$ & 2.10 & - & $1.00 \mathrm{E}+17$ & 2,9 \\
\hline $\mathrm{Cu} 2 \mathrm{O}$ & 2.20 & - & $1.00 \mathrm{E}+15$ & $28,92-94$ \\
\hline $\mathrm{LaCuOTe}$ & 2.20 & - & $1.30 \mathrm{E}+17$ & 95 \\
\hline
\end{tabular}




\begin{tabular}{|c|c|c|c|c|}
\hline $\mathrm{ZnTe}$ & 2.25 & - & $1.00 \mathrm{E}+18$ & 96 \\
\hline $\mathrm{Cu}_{3} \mathrm{PS}_{4}$ & 2.38 & - & $1.80 \mathrm{E}+17$ & 2 \\
\hline $\mathrm{CuGaS}_{2}$ & 2.38 & - & $5.40 \mathrm{E}+17$ & 2 \\
\hline $\mathrm{CuAlS}_{2}$ & 2.50 & - & $1.00 \mathrm{E}+19$ & 2 \\
\hline $\mathrm{CuGaO}_{2}$ & 2.60 & - & $1.70 \mathrm{E}+18$ & 17 \\
\hline $\mathrm{CuAlSe}_{2}$ & 2.67 & - & $1.00 \mathrm{E}+18$ & 2 \\
\hline LaCuOSe & 2.80 & - & $1.00 \mathrm{E}+20$ & 95,97 \\
\hline $\mathrm{ZnIn}_{2} \mathrm{~S}_{4}$ & 2.90 & - & $1.00 \mathrm{E}+17$ & 2 \\
\hline $\mathrm{CuI}$ & 2.95 & - & $1.00 \mathrm{E}+20$ & $2,98,99$ \\
\hline $\mathrm{CuAlO}_{2}$ & 2.97 & - & $1.30 \mathrm{E}+17$ & 100,101 \\
\hline $\mathrm{LaCuOS}$ & 3.10 & - & $1.00 \mathrm{E}+19$ & 95,97 \\
\hline $\mathrm{SrCu}_{2} \mathrm{O}_{2}$ & 3.30 & - & $1.00 \mathrm{E}+17$ & 102,103 \\
\hline
\end{tabular}




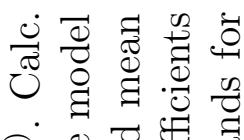

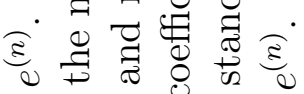

+ घ्ञ 되

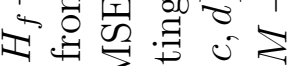

ব ठृ

$\times \underbrace{0}_{0}$

हิ $\frac{\pi}{3}: 0.0$

$+\frac{0}{\sigma} 00 . \bar{\Xi}$

w 0 o

$\times \frac{\vec{J}}{\vec{Z}}$ 击

हु

1.0

¿

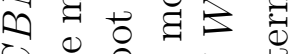

U

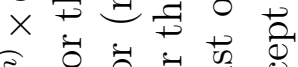

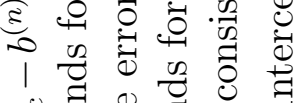

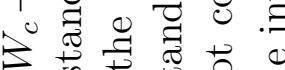

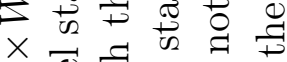

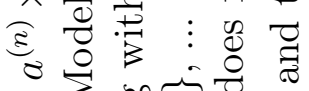

$\| \sum_{\sigma}^{\infty} \overbrace{0}^{\infty} \approx$

हि

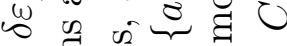

.

可

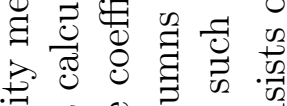

宩

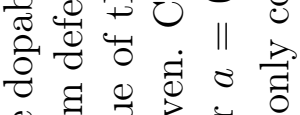

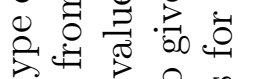

id

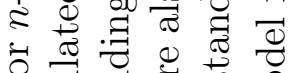

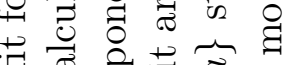

$=\begin{array}{cccc}0 & 0 & 0 & 0 \\ 0 & 0 & 0 & 0\end{array}$

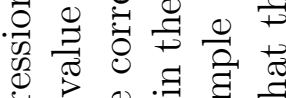

क人⿻

可

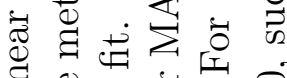

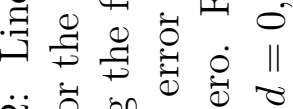

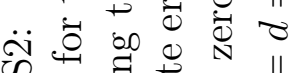

记

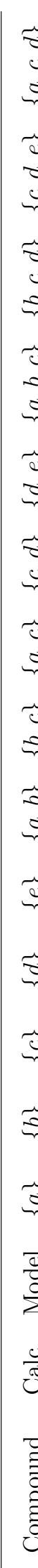

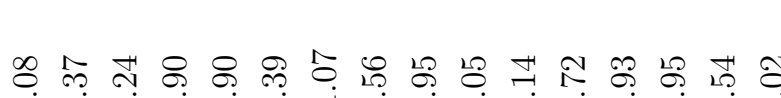

-

농 우 ᄋ จ

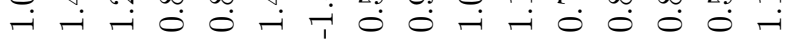

됴 $\frac{6}{0}$.

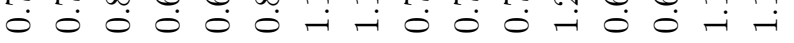

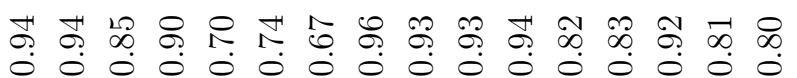

邑

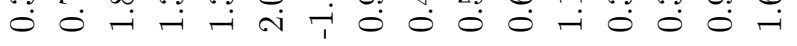

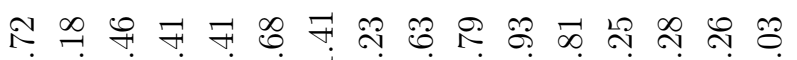

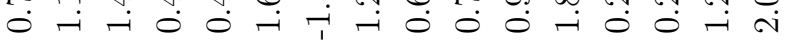

\& ఝ

虽

ஜ

00000001000100

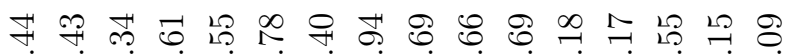

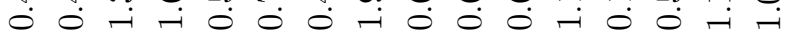

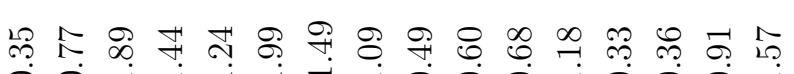

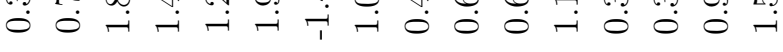

ஐ

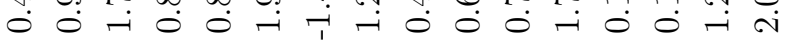

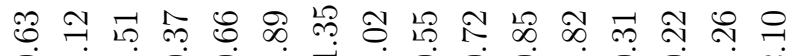

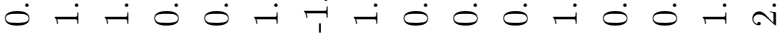

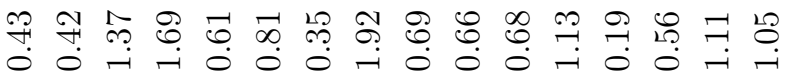

๓ึ่

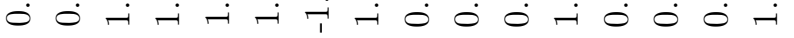

भ

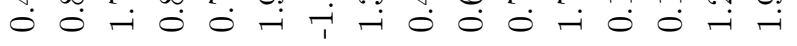

ำ 궁

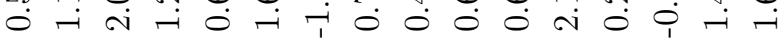

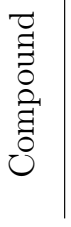

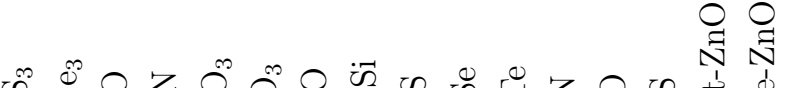

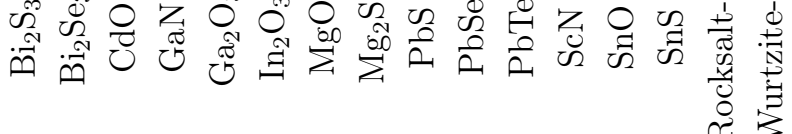

\begin{tabular}{|c|c|}
\hline 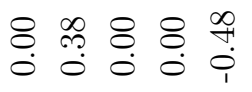 & 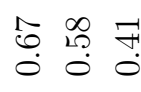 \\
\hline 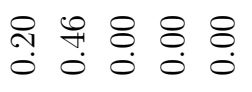 & 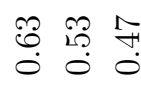 \\
\hline 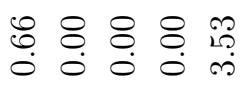 & $\begin{array}{lll}\overrightarrow{1} & 0 & 0 \\
\infty & 0 & 0 \\
0 & 0 & 0 \\
0 & 0 & 0\end{array}$ \\
\hline $\begin{array}{lllll}\mathbb{8} & \mathbb{8} & \mathbb{2} & \infty \\
\stackrel{0}{0} & \stackrel{0}{0} & \stackrel{0}{0} & \stackrel{0}{0}\end{array}$ & $\begin{array}{lll}0 & \infty & 0 \\
\infty & 0 & 0 \\
0 & 0 & 0\end{array}$ \\
\hline \begin{tabular}{lllll}
0 & 20 & 8 & 8 & 8 \\
\hdashline & 10 & 0 & 0 \\
0 & 0 & 0 & 0 & 0
\end{tabular} & 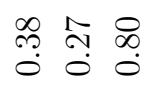 \\
\hline 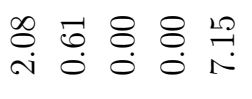 & 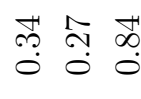 \\
\hline 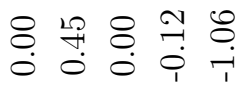 & 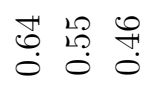 \\
\hline 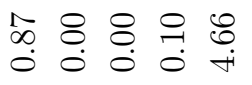 & 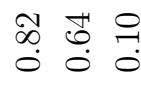 \\
\hline 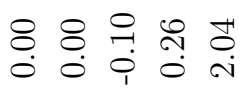 & 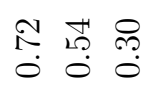 \\
\hline 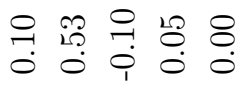 & 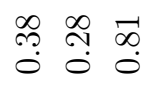 \\
\hline 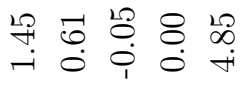 & $\begin{array}{lll}\stackrel{\imath}{N} & \bar{\sigma} & \bar{\sigma} \\
0 & 0 & 0\end{array}$ \\
\hline 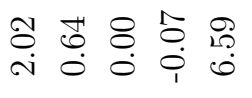 & 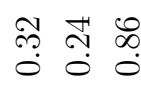 \\
\hline 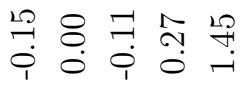 & $\begin{array}{lll}0 & 0 \\
0 & 0 & 0 \\
0 & 0 & 0 \\
0\end{array}$ \\
\hline 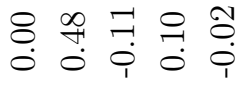 & \begin{tabular}{ccc}
9 & \multirow{1}{*}{} & 0 \\
0 & 0 & 0 \\
0
\end{tabular} \\
\hline 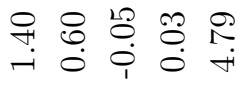 & $\begin{array}{lll}\stackrel{N}{N} & \vec{N} & \vec{\sigma} \\
0 & 0 & 0\end{array}$ \\
\hline 0000 & 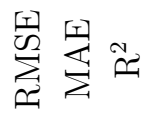 \\
\hline
\end{tabular}

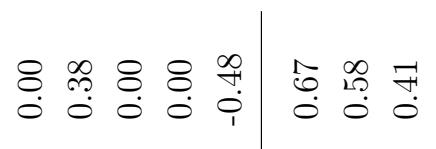

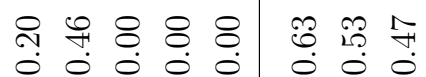

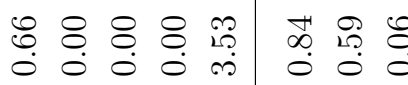

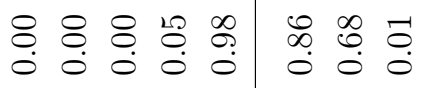

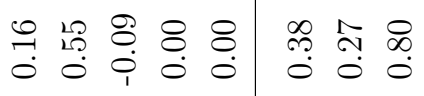

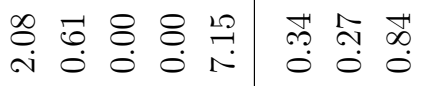

\&

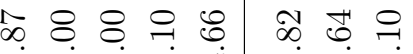

$88 ㅇ ㅠ$

으

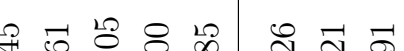

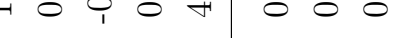




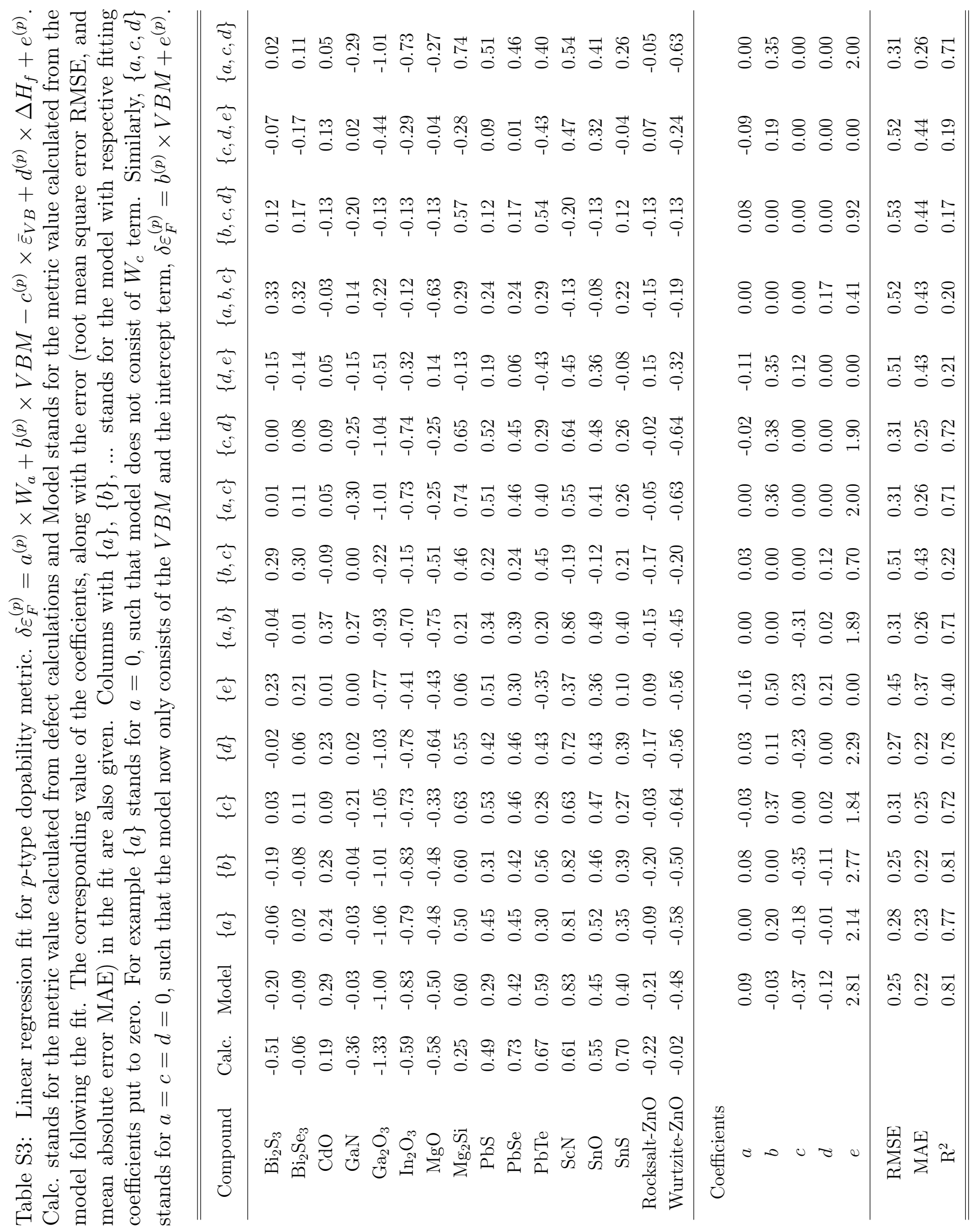


Table S4: Calculated cation vacancy formation energy $\Delta \mathrm{H}$ at the conduction band minimum $(C B M)$ and the $n$-type pinning energy $\varepsilon_{F}^{(n)}$ relative to the vacuum, along with the charge state $q$. In the model cation-rich conditions are assumed.

\begin{tabular}{ccccc}
\hline \hline Compound & Defect $(D)$ & Charge $(q)$ & $\Delta \mathrm{H}(D, q) @ \mathrm{CBM}$ & $\varepsilon_{F}^{(n)}$ \\
\hline $\mathrm{Bi}_{2} \mathrm{~S}_{3}$ & $\mathrm{~V}_{\mathrm{Bi}}$ & -3 & 1.55 & -3.65 \\
$\mathrm{Bi}_{2} \mathrm{Se}_{3}$ & $\mathrm{~V}_{\mathrm{Bi}}$ & -3 & 3.05 & -3.81 \\
$\mathrm{CdO}$ & $\mathrm{V}_{\mathrm{Cd}}$ & -2 & 4.08 & -2.86 \\
$\mathrm{GaN}$ & $\mathrm{V}_{\mathrm{Ga}}$ & -3 & 3.73 & -2.28 \\
$\mathrm{Ga}_{2} \mathrm{O}_{3}$ & $\mathrm{~V}_{\mathrm{Ga}}$ & -3 & 1.84 & -3.09 \\
$\mathrm{In}_{2} \mathrm{O}_{3}$ & $\mathrm{~V}_{\mathrm{In}}$ & -3 & 6.35 & -3.35 \\
$\mathrm{MgO}$ & $\mathrm{V}_{\mathrm{Mg}}$ & -2 & -2.85 & -0.54 \\
$\mathrm{Mg}{ }_{2} \mathrm{Si}$ & $\mathrm{V}_{\mathrm{Mg}}$ & -2 & 1.58 & -1.99 \\
$\mathrm{PbS}$ & $\mathrm{V}_{\mathrm{Pb}}$ & -2 & 0.93 & -3.36 \\
$\mathrm{PbSe}$ & $\mathrm{V}_{\mathrm{Pb}}$ & -2 & 1.27 & -3.46 \\
$\mathrm{PbTe}$ & $\mathrm{V}_{\mathrm{Pb}}$ & -2 & 1.26 & -3.77 \\
$\mathrm{ScN}$ & $\mathrm{V}_{\mathrm{Sc}}$ & -3 & 6.48 & -1.04 \\
$\mathrm{SnO}$ & $\mathrm{V}_{\mathrm{Sn}}$ & -2 & 0.40 & -3.57 \\
$\mathrm{SnS}$ & $\mathrm{V}_{\mathrm{Sn}}$ & -2 & -0.20 & -3.93 \\
$\mathrm{Rocksalt} \mathrm{ZnO}$ & $\mathrm{V}_{\mathrm{Zn}}$ & -2 & 2.87 & -1.98 \\
Wurtzite ZnO & $\mathrm{V}_{\mathrm{Zn}}$ & -2 & 3.36 & -2.68 \\
\hline \hline
\end{tabular}


Table S5: Calculated anion vacancy formation energy $\Delta \mathrm{H}$ at the valence band maximum $(V B M)$ and the $p$-type pinning energy $\varepsilon_{F}^{(p)}$ relative to the vacuum, along with the charge state, $q$. In the model anion-rich conditions are assumed. For $\mathrm{Mg}_{2} \mathrm{Si}$, the lowest energy intrinsic donor is cation interstitial instead of the anion vacancy.

\begin{tabular}{ccccc}
\hline \hline \multirow{2}{*}{ Compound } & Defect $(D)$ & Charge $(q)$ & $\Delta \mathrm{H}(D, q) @ \mathrm{VBM}$ & $\varepsilon_{F}^{(p)}$ \\
\hline $\mathrm{Bi}_{2} \mathrm{~S}_{3}$ & $\mathrm{~V}_{\mathrm{S}}$ & 2 & -1.02 & -5.08 \\
$\mathrm{Bi}_{2} \mathrm{Se}_{3}$ & $\mathrm{~V}_{\mathrm{Se}}$ & 2 & -0.11 & -5.27 \\
$\mathrm{CdO}$ & $\mathrm{V}_{\mathrm{O}}$ & 2 & 0.37 & -5.89 \\
$\mathrm{GaN}$ & $\mathrm{V}_{\mathrm{N}}$ & 3 & -1.07 & -6.61 \\
$\mathrm{Ga}_{2} \mathrm{O}_{3}$ & $\mathrm{~V}_{\mathrm{O}}$ & 2 & -2.66 & -7.17 \\
$\mathrm{In}_{2} \mathrm{O}_{3}$ & $\mathrm{~V}_{\mathrm{O}}$ & 2 & -1.18 & -7.13 \\
$\mathrm{MgO}$ & $\mathrm{V}_{\mathrm{O}}$ & 2 & -1.15 & -6.72 \\
$\mathrm{Mg}{ }_{2} \mathrm{Si}$ & $\mathrm{Mg}$ & 2 & -0.67 & -3.21 \\
$\mathrm{PbS}$ & $\mathrm{V}_{\mathrm{S}}$ & 2 & 0.97 & -4.70 \\
$\mathrm{PbSe}$ & $\mathrm{V}_{\mathrm{Se}}$ & 2 & 1.45 & -5.08 \\
$\mathrm{PbTe}$ & $\mathrm{V}_{\mathrm{Te}}$ & 2 & 1.33 & -5.18 \\
$\mathrm{ScN}$ & $\mathrm{V}_{\mathrm{N}}$ & 3 & 1.84 & -4.73 \\
$\mathrm{SnO}$ & $\mathrm{V}_{\mathrm{O}}$ & 2 & 1.10 & -5.05 \\
$\mathrm{SnS}$ & $\mathrm{V}_{\mathrm{S}}$ & 2 & 1.40 & -5.60 \\
$\mathrm{Rocksalt} \mathrm{ZnO}$ & $\mathrm{V}_{\mathrm{O}}$ & 2 & -0.44 & -6.59 \\
Wurtzite $\mathrm{ZnO}$ & $\mathrm{V}_{\mathrm{O}}$ & 2 & -0.03 & -7.72 \\
\hline \hline
\end{tabular}


Table S6: Reference phase ionization energy (for gas phase) and work function (for solid phase) with respect to the vacuum, obtained from Refs. ${ }^{54,104}$ Cohesive energy of a solid or gas (molecule) phase is the energy required to break it into its constituent gas phase, and is obtained from page 50 of Ref. ${ }^{105}$

\begin{tabular}{|c|c|c|c|c|c|}
\hline \multirow{2}{*}{$\begin{array}{c}\text { Reference } \\
\text { phase }\end{array}$} & \multicolumn{3}{|c|}{ Ionization Energy or Work function } & \multicolumn{2}{|c|}{ Cohesive Energy } \\
\hline & $(\mathrm{eV})$ & Method & Additional Details & $(\mathrm{kJ} / \mathrm{mol})$ & in $\mathrm{eV} /$ atom \\
\hline $\mathrm{Bi}(\mathrm{s})$ & -4.34 & Photoelectric effect & Polycrystalline sample & -210.0 & -2.18 \\
\hline $\mathrm{Cd}(\mathrm{s})$ & -4.08 & Contact potential difference & Polycrystalline sample & -112.0 & -1.16 \\
\hline $\mathrm{Ga}(\mathrm{s})$ & -4.32 & Photoelectric effect & Polycrystalline sample & -271.0 & -2.81 \\
\hline In $(s)$ & -4.09 & Photoelectric effect & Polycrystalline sample & -243.0 & -2.52 \\
\hline $\operatorname{Mg}(\mathrm{s})$ & -3.66 & Photoelectric effect & Polycrystalline sample & -145.0 & -1.51 \\
\hline $\mathrm{N}_{2}(\mathrm{~g})$ & -14.53 & & & -945.0 & -4.90 \\
\hline $\mathrm{O}_{2}(\mathrm{~g})$ & -13.62 & & & -498.0 & -2.58 \\
\hline $\mathrm{Pb}(\mathrm{s})$ & -4.25 & Photoelectric effect & Polycrystalline sample & -196.0 & -2.03 \\
\hline $\mathrm{S}(\mathrm{s})$ & -10.36 & & & -275.0 & -2.85 \\
\hline $\mathrm{Sb}(\mathrm{s})$ & -4.70 & Photoelectric effect & 100 plane & -265.0 & -2.75 \\
\hline $\mathrm{Sc}(\mathrm{s})$ & -3.50 & Photoelectric effect & Polycrystalline sample & -376.0 & -3.90 \\
\hline Se $(s)$ & -5.90 & Photoelectric effect & Polycrystalline sample & -237.0 & -2.46 \\
\hline $\mathrm{Si}(\mathrm{s})$ & -4.60 & Photoelectric effect & 111 plane & -446.0 & -4.63 \\
\hline $\mathrm{Si}(\mathrm{s})$ & -4.91 & Contact potential difference & 100 plane & & \\
\hline $\operatorname{Sn}(\mathrm{s})$ & -4.42 & Contact potential difference & Polycrystalline sample & -303.0 & -3.14 \\
\hline $\mathrm{Te}(\mathrm{s})$ & -4.95 & Photoelectric effect & Polycrystalline sample & -211.0 & -2.19 \\
\hline $\mathrm{Zn}(\mathrm{s})$ & -3.63 & Photoelectric effect & Polycrystalline sample & -130.0 & -1.35 \\
\hline $\mathrm{Zn}(\mathrm{s})$ & -4.90 & Contact potential difference & Polycrystalline sample & & \\
\hline
\end{tabular}




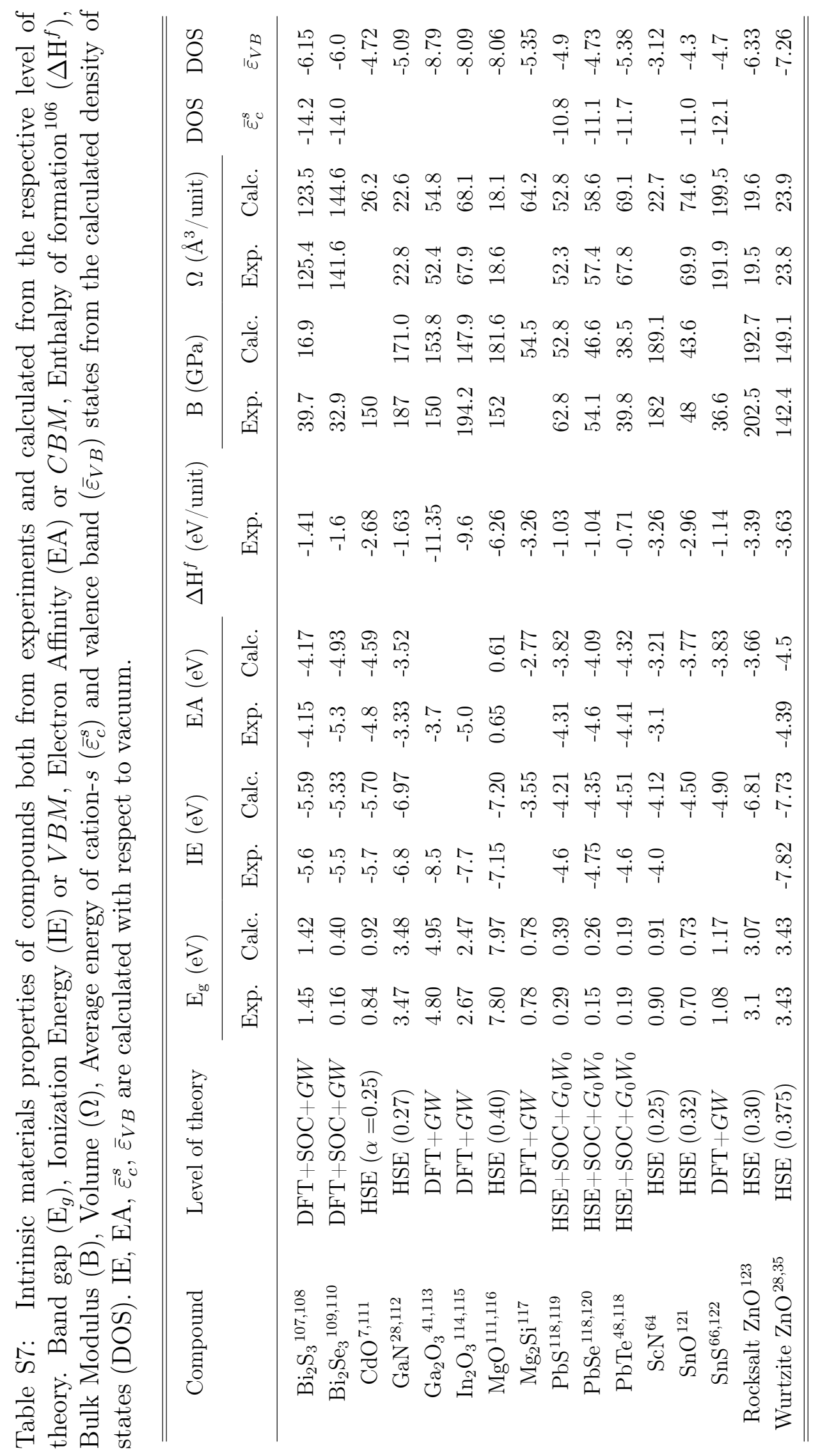




\section{References}

(1) Gorai, P.; Goyal, A.; Toberer, E. S.; Stevanović, V. A simple chemical guide for finding novel n-type dopable Zintl pnictide thermoelectric materials. Journal of Materials Chemistry A 2019, 7, 19385-19395.

(2) Miller, S. A.; Dylla, M.; Anand, S.; Gordiz, K.; Snyder, G. J.; Toberer, E. S. Empirical modeling of dopability in diamond-like semiconductors. npj Computational Materials 2018, $\underline{4}, 71$.

(3) Yu, Y. G.; Zhang, X.; Zunger, A. Natural off-stoichiometry causes carrier doping in half-Heusler filled tetrahedral structures. Physical Review B 2017, 95, 085201.

(4) Anderson, P. A. Indium Nitride: An Investigation of Growth, Electronic Structure and Doping. Ph.D. thesis, University of Canterbury, 2006.

(5) Sachet, E.; Shelton, C. T.; Harris, J. S.; Gaddy, B. E.; Irving, D. L.; Curtarolo, S.; Donovan, B. F.; Hopkins, P. E.; Sharma, P. A.; Sharma, A. L.; Ihlefeld, J.; Franzen, S.; Maria, J.-P. Dysprosium-doped cadmium oxide as a gateway material for mid-infrared plasmonics. Nature Materials 2015, 14, 414-420.

(6) Dou, Y.; Egdell, R.; Walker, T.; Law, D.; Beamson, G. N-type doping in CdO ceramics: a study by EELS and photoemission spectroscopy. Surface Science 1998, 398, 241258.

(7) Burbano, M.; Scanlon, D. O.; Watson, G. W. Sources of Conductivity and Doping Limits in $\mathrm{CdO}$ from Hybrid Density Functional Theory. Journal of the American Chemical Society 2011, 133, 15065-15072.

(8) Zinc arsenide (ZnAs2) resistivity, carrier concentration and mobility, thermoelectric power and thermal conductivity: Datasheet from Landolt-Börnstein - Group 
III Condensed Matter • Volume 41C: "Non-Tetrahedrally Bonded Elements and Binary Compounds I" in. https://materials.springer.com/lb/docs/sm\{\_\}Ibs $\left\{\backslash_{\text {_ }}\right.$ \}978-3-540-31360-1\{\_\}296.

(9) Martinez, A. D.; Fioretti, A. N.; Toberer, E. S.; Tamboli, A. C. Synthesis, structure, and optoelectronic properties of II-IV-V 2 materials. Journal of Materials Chemistry A 2017, 5, 11418-11435.

(10) CdAs2 rt charge carrier concentration: Datasheet from "PAULING FILE Multinaries Edition - 2012" in SpringerMaterials. https://materials.springer.com/isp/ physical-property/docs/ppp\{\_\}2798f764b55658e860c4c62408f637f9.

(11) Biswas, K.; Zhao, L.-D.; Kanatzidis, M. G. Tellurium-Free Thermoelectric: The Anisotropic n-Type Semiconductor Bi2S3. Advanced Energy Materials 2012, 2, 634638.

(12) Bi2S3 charge carrier concentration: Datasheet from "PAULING FILE Multinaries Edition - 2012" in Spr ingerMaterials. https://materials.springer.com/isp/ physical-property/docs/ppp\{\_\}4c819d3b38b28edc32d590df4441cd18.

(13) Yu, D. n-Type Conducting CdSe Nanocrystal Solids. Science 2003, 300, 1277-1280.

(14) Sze, S.; Ng, K. K. Physics of Semiconductor Devices; John Wiley \& Sons, Inc.: Hoboken, NJ, USA, 2006.

(15) Sb2S3 energy gap: Datasheet from "PAULING FILE Multinaries Edition - 2012" in SpringerMaterials. https://materials.springer.com/isp/physical-property/ docs/ppp\{\_\}2ecbfb3a11855a7587d8051fc68d778f.

(16) Ibuki, S.; Yanagi, H.; Ueda, K.; Kawazoe, H.; Hosono, H. Preparation of n -type conductive transparent thin films of AgInO2:Sn with delafossite-type structure by pulsed laser deposition. Journal of Applied Physics 2000, 88, 3067-3069. 
(17) Kumar, M.; Zhao, H.; Persson, C. Study of band-structure, optical properties and native defects in A I B III O 2 ( $\mathrm{A} \mathrm{I}=\mathrm{Cu}$ or $\mathrm{Ag}$, B III $=\mathrm{Al}$, Ga or In) delafossites. Semiconductor Science and Technology 2013, 28, 065003.

(18) Kumaravel, R.; Ramamurthi, K. Structural, optical and electrical properties of In-doped Cd2SnO4 thin films by spray pyrolysis method. Journal of Alloys and Compounds 2011, 509, 4390-4393.

(19) $\mathrm{Cd} 2 \mathrm{SnO} 4$ rt charge carrier concentration: Datasheet from "PAULING FILE Multinaries Edition - 2012" in SpringerMaterials. https://materials.springer.com/isp/ physical-property/docs/ppp\{\_\}0e21621053d5c7fa81f5fce26ac674f7.

(20) Segev, D.; Wei, S.-H. Structure-derived electronic and optical properties of transparent conducting oxides. Physical Review B 2005, 71, 125129.

(21) Colston, G.; Myronov, M. Electrical properties of n-type 3C-SiC epilayers in situ doped with extremely high levels of phosphorus. Semiconductor Science and Technology 2018, 33, 114007.

(22) $\mathrm{SiC} 3 \mathrm{C}$ charge carrier concentration: Datasheet from "PAULING FILE Multinaries Edition - 2012" in Sp ringerMaterials. https://materials.springer.com/isp/ physical-property/docs/ppp\{\_\}e369e6c5cbdc9d2f3f703e5c77ae2652.

(23) Aluminum phosphide (AlP), electrical and thermal transport: Datasheet from Landolt-Börnstein - Group III Condensed Matter Volume 41A1: "Group IV Elements, IV-IV and III-V Compounds. Part b - Electronic, Transport, Optical and Other Properties" in Sprin. https://materials.springer.com/lb/docs/sm\{ $\backslash_{-}$ \}lbs $\left\{\backslash_{-}\right\} 978-3-540-31356-4\left\{\backslash_{-}\right\} 67$.

(24) Crandall, R. S. Electrical Conduction in n-Type Cadmium Sulfide at Low Temperatures. Physical Review 1968, 169, 577-584. 
(25) Limpijumnong, S.; Reunchan, P.; Janotti, A.; Van De Walle, C. G. Hydrogen doping in indium oxide: An ab initio study. Physical Review B - Condensed Matter and Materials Physics 2009, 80, 1-4.

(26) Walsh, A.; Da Silva, J. L. F.; Wei, S.-H.; Körber, C.; Klein, A.; Piper, L. F. J.; DeMasi, A.; Smith, K. E.; Panaccione, G.; Torelli, P.; Payne, D. J.; Bourlange, A.; Egdell, R. G. Physical Review Letters 2008, 100, 167402.

(27) Bally, A. Electronic properties of nano-crystalline titanium dioxide thin films. Ph.D. thesis, 1999.

(28) Stevanović, V.; Lany, S.; Ginley, D. S.; Tumas, W.; Zunger, A. Assessing capability of semiconductors to split water using ionization potentials and electron affinities only. Physical chemistry chemical physics : PCCP 2014, 16, 3706-14.

(29) Kim, H. J.; Kim, U.; Kim, T. H.; Kim, J.; Kim, H. M.; Jeon, B.-G.; Lee, W.-J.; Mun, H. S.; Hong, K. T.; Yu, J.; Char, K.; Kim, K. H. Physical Review B 2012, $\underline{86}$, 165205.

(30) Tufte, O. N.; Chapman, P. W. Electron Mobility in Semiconducting Strontium Titanate. Physical Review 1967, 155, 796-802.

(31) van Benthem, K.; Elsässer, C.; French, R. H. Bulk electronic structure of SrTiO3: Experiment and theory. Journal of Applied Physics 2001, 90, 6156-6164.

(32) Dimitrievska, M.; Ivetić, T. B.; Litvinchuk, A. P.; Faairbrother, A.; Miljević, B. B.; Štrbac, G. R.; Pérez Rodríguez, A.; Lukić-Petrović, S. R. Eu 3+ -Doped Wide Band Gap Zn $2 \mathrm{SnO} 4$ Semiconductor Nanoparticles: Structure and Luminescence. The Journal of Physical Chemistry C 2016, 120, 18887-18894.

(33) Kawazoe, H.; Ueda, K. Transparent Conducting Oxides Based on the Spinel Structure. Journal of the American Ceramic Society 2004, 82, 3330-3336. 
(34) Ueda, N.; Omata, T.; Hikuma, N.; Ueda, K.; Mizoguchi, H.; Hashimoto, T.; Kawazoe, H. New oxide phase with wide band gap and high electroconductivity, MgIn 2 O 4. Applied Physics Letters 1992, 61, 1954-1955.

(35) Özgür, Ü.; Alivov, Y. I.; Liu, C.; Teke, A.; Reshchikov, M. A.; Doğan, S.; Avrutin, V.; Cho, S.-J.; Morkoç, H. A comprehensive review of $\mathrm{ZnO}$ materials and devices. Journal of Applied Physics 2005, 98, 1-103.

(36) Edwards, P. P.; Porch, A.; Jones, M. O.; Morgan, D. V.; Perks, R. M. Basic materials physics of transparent conducting oxides. Dalton Transactions 2004, 2995.

(37) Nagasawa, M.; Shionoya, S. Exciton structure in optical absorption of SnO2 crystals. Physics Letters 1966, 22, 409-410.

(38) Hadjarab, B.; Bouguelia, A.; Trari, M. Synthesis, physical and photo electrochemical characterization of La-doped SrSnO3. Journal of Physics and Chemistry of Solids 2007, $\underline{68}, 1491-1499$.

(39) Bellal, B.; Hadjarab, B.; Bouguelia, A.; Trari, M. Visible light photocatalytic reduction of water using $\mathrm{SrSnO} 3$ sensitized by $\mathrm{CuFeO}$. Theoretical and Experimental Chemistry 2009, 45, 172-179.

(40) Lany, S. Defect phase diagram for doping of Ga2O3. APL Materials 2018, $\underline{6}$.

(41) Pearton, S. J.; Yang, J.; Cary, P. H.; Ren, F.; Kim, J.; Tadjer, M. J.; Mastro, M. A. A review of Ga 2 O 3 materials, processing, and devices. Applied Physics Reviews 2018, $\underline{5}, 011301$.

(42) Yashima, I.; Watanave, H.; Ogisu, T.; Tsukuda, R.; Sat̃o, S. Thermoelectric Properties and Hall Effect of Bi 2 Te 3 - x Se x Polycrystalline Materials Prepared by a Hot Press Method. Japanese Journal of Applied Physics 1998, 37, 2472-2473. 
(43) Hor, Y. S.; Richardella, A.; Roushan, P.; Xia, Y.; Checkelsky, J. G.; Yazdani, A.; Hasan, M. Z.; Ong, N. P.; Cava, R. J. Physical Review B 2009, 79, 195208.

(44) Li, M.; Wang, Z.; Yang, L.; Li, D.; Yao, Q. R.; Rao, G. H.; Gao, X. P. A.; Zhang, Z. Physical Review B 2017, 96, 075152.

(45) Filip, M. R.; Patrick, C. E.; Giustino, F. Physical Review B 2013, 87, 205125.

(46) Levinshtein, $\quad$ M.; $\quad$ Rumyantsev, $\quad$ S. $\quad$ L.; $\quad$ Shur, $\quad$ M. Handbook series on semiconductor parameters. Vol. 1, Si, Ge, C (diamond), GaAs, GaP, GaSb, In Singapore ; New Jersey : World Scientific, 1996.

(47) PbSe charge carrier concentration: Datasheet from "PAULING FILE Multinaries Edition - 2012" in Spri ngerMaterials. https://materials.springer.com/isp/ physical-property/docs/ppp\{\_\}7ec1c948a29528b9acf9cb40911ea701.

(48) Goyal, A.; Gorai, P.; Toberer, E. S.; Stevanović, V. First-principles calculation of intrinsic defect chemistry and self-doping in PbTe. npj Computational Materials 2017, 3, 42 .

(49) Male, J.; Agne, M. T.; Goyal, A.; Anand, S.; Witting, I. T.; Stevanović, V.; Snyder, G. J. The importance of phase equilibrium for doping efficiency: iodine doped PbTe. Materials Horizons 2019,

(50) PbS charge carrier concentration: Datasheet from "PAULING FILE Multinaries Edition - 2012" in Sp ringerMaterials. https://materials.springer.com/isp/ physical-property/docs/ppp\{\_\}45dbe0d0b9aad1149f9773f6b8ef8316.

(51) Ohno, S.; Imasato, K.; Anand, S.; Tamaki, H.; Kang, S. D.; Gorai, P.; Sato, H. K.; Toberer, E. S.; Kanno, T.; Snyder, G. J. Phase Boundary Mapping to Obtain n-type Mg3Sb2-Based Thermoelectrics. Joule 2018, 2, 141-154. 
(52) Shuai, J.; Wang, Y.; Kim, H. S.; Liu, Z.; Sun, J.; Chen, S.; Sui, J.; Ren, Z. Thermoelectric properties of Na-doped Zintl compound: Mg3NaSb2. Acta Materialia 2015, 93, $187-193$.

(53) Zhang, J.; Song, L.; Pedersen, S. H.; Yin, H.; Hung, L. T.; Iversen, B. B. Discovery of high-performance low-cost n-type Mg3Sb2-based thermoelectric materials with multivalley conduction bands. Nature Communications 2017, $\underline{8}, 13901$.

(54) Lide R., D., Ed. CRC Handbook of Chemistry and Physics, 9th ed.; 2009; pp 12-114.

(55) Hosono, H.; Ogo, Y.; Yanagi, H.; Kamiya, T. Bipolar Conduction in SnO Thin Films. Electrochemical and Solid-State Letters 2011, 14, H13.

(56) Strauss, A. J. Physical Review 1961, 121, 1087-1090.

(57) Mitchel, W. C.; Elhamri, S.; Haugan, H. J.; Berney, R.; Mou, S.; Brown, G. J. Electrical properties of n-type GaSb substrates and p-type GaSb buffer layers for InAs/InGaSb superlattice infrared detectors. AIP Advances 2015, 5, 097219.

(58) Nolas, G. S.; Wang, D.; Beekman, M. Transport properties of polycrystalline Mg2Si1ySby. Physical Review B 2007, 76, 235204.

(59) Kolezynski, A.; Nieroda, P.; Wojciechowski, K. T. Li doped Mg2Si p-type thermoelectric material: Theoretical and experimental study. Computational Materials Science 2015, 100, 84-88.

(60) SnSe rt charge carrier concentration: Datasheet from "PAULING FILE Multinaries Edition - 2012" i n SpringerMaterials. https://materials.springer.com/isp/ physical-property/docs/ppp\{\_\}524046fdf85db860ef88122fcba273a8.

(61) Duong, A. T.; Nguyen, V. Q.; Duvjir, G.; Duong, V. T.; Kwon, S.; Song, J. Y.; Lee, J. K.; Lee, J. E.; Park, S.; Min, T.; Lee, J.; Kim, J.; Cho, S. Achieving ZT=2.2 with Bi-doped n-type SnSe single crystals. Nature Communications 2016, ㄱ, 13713. 
(62) Deng, R.; Ozsdolay, B. D.; Zheng, P. Y.; Khare, S. V.; Gall, D. Optical and transport measurement and first-principles determination of the ScN band gap. Physical Review B 2015, 91, 045104.

(63) Saha, B.; Garbrecht, M.; Perez-Taborda, J. A.; Fawey, M. H.; Koh, Y. R.; Shakouri, A.; Martin-Gonzalez, M.; Hultman, L.; Sands, T. D. Compensation of native donor doping in ScN: Carrier concentration control and p -type ScN. Applied Physics Letters 2017, $110,252104$.

(64) Kumagai, Y.; Tsunoda, N.; Oba, F. Point Defects and p-Type Doping in ScN from First Principles. Physical Review Applied 2018, 9, 34019.

(65) Fioretti, A. N.; Schwartz, C. P.; Vinson, J.; Nordlund, D.; Prendergast, D.; Tamboli, A. C.; Caskey, C. M.; Tuomisto, F.; Linez, F.; Christensen, S. T.; Toberer, E. S.; Lany, S.; Zakutayev, A. Understanding and control of bipolar self-doping in copper nitride. Journal of Applied Physics 2016, 119, 181508.

(66) Vidal, J.; Lany, S.; D’Avezac, M.; Zunger, A.; Zakutayev, A.; Francis, J.; Tate, J. Bandstructure, optical properties, and defect physics of the photovoltaic semiconductor SnS. Applied Physics Letters 2012, 100.

(67) Xiao, Z.; Ran, F.-Y.; Hosono, H.; Kamiya, T. Route to n -type doping in SnS. Applied

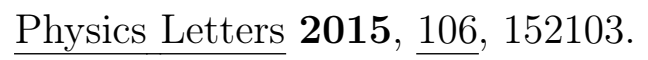

(68) Bugajski, M.; Lewandowski, W. Concentration-dependent absorption and photoluminescence of $\mathrm{n}$-type InP. Journal of Applied Physics 1985, 57, 521-530.

(69) Neave, J. H.; Dobson, P. J.; Harris, J. J.; Dawson, P.; Joyce, B. A. Silicon doping of MBE-grown GaAs films. Applied Physics A Solids and Surfaces 1983, 32, 195-200.

(70) Sun, S.; Armour, E.; Zheng, K.; Schaus, C. Zinc and tellurium doping in GaAs and AlGaAs grown by MOCVD. Journal of Crystal Growth 1991, 113, 103-112. 
(71) Yanagi, H.; Hase, T.; Ibuki, S.; Ueda, K.; Hosono, H. Bipolarity in electrical conduction of transparent oxide semiconductor $\mathrm{CuInO} 2$ with delafossite structure. Applied Physics Letters 2001, 78, 1583-1585.

(72) Segall, B.; Lorenz, M. R.; Halsted, R. E. Physical Review 1963, 129, 2471-2481.

(73) Perrenoud, J.; Kranz, L.; Gretener, C.; Pianezzi, F.; Nishiwaki, S.; Buecheler, S.; Tiwari, A. N. A comprehensive picture of $\mathrm{Cu}$ doping in CdTe solar cells. Journal of

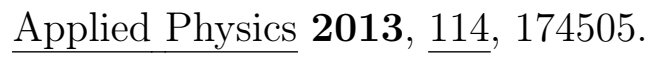

(74) Nagaoka, A.; Kuciauskas, D.; McCoy, J.; Scarpulla, M. A. High p-type doping, mobility, and photocarrier lifetime in arsenic-doped CdTe single crystals. Applied Physics Letters 2018, 112, 192101.

(75) Su, C. H. Energy band gap, intrinsic carrier concentration, and Fermi level of CdTe bulk crystal between 304 and 1067 K. Journal of Applied Physics 2008, 103.

(76) Aluminum arsenide (AlAs), electrical and thermal conductivity, carrier concentration: Datasheet from Landolt-Börnstein - Group III Condensed Matter Volume 41A1: "Group IV Elements, IV-IV and III-V Compounds. Part b - Electronic, Transport, Optical an. https://materials.springer.com/lb/docs/sm\{\_\}lbs $\left\{\backslash_{-}\right.$ 3978-3-540-31356-4\{\_\}84.

(77) GaP rt charge carrier concentration: Datasheet from "PAULING FILE Multinaries Edition - 2012" in SpringerMaterials. https://materials.springer.com/isp/ physical-property/docs/ppp\{\_\}5215e83ea64ca9960e3ef91c61d54fb3.

(78) Simpson, J.; Wallace, J. M.; Wang, S. Y.; Stewart, H.; Hunter, J. J.; Adams, S. J.; Prior, K. A.; Cavenett, B. C. N-type doping of molecular beam epitaxial zinc selenide using an electrochemical iodine cell. Semiconductor Science and Technology 1992, $\underline{7}$, 464-466. 
(79) Van De Walle, C. G.; Laks, D. B.; Neumark, G. F.; Pantelides, S. T. First-principles calculations of solubilities and doping limits: Li, Na, and $\mathrm{N}$ in ZnSe. Physical Review B 1993, $47,9425-9434$.

(80) Ueno, K.; Fudetani, T.; Arakawa, Y.; Kobayashi, A.; Ohta, J.; Fujioka, H. Electron transport properties of degenerate n-type GaN prepared by pulsed sputtering. APL Materials 2017, $\underline{5}, 126102$.

(81) Tsu, R.; Howard, W. E.; Esaki, L. Optical and Electrical Properties and Band Structure of GeTe and SnTe. Physical Review 1968, 172, 779-788.

(82) Sb2Te3 charge carrier concentration: Datasheet from "PAULING FILE Multinaries Edition - 2012" in SpringerMaterials. https://materials.springer.com/isp/ physical-property/docs/ppp\{\_\}1bc8778115586bd2ca54f5aebe46fdf8.

(83) Tenga, A.; Lidin, S.; Belieres, J.-P.; Newman, N.; Wu, Y.; Haeussermann, U. ChemInform Abstract: Metastable Cd 4 Sb 3 : A Complex Structured Intermetallic Compound with Semiconductor Properties. ChemInform 2009, 40, 15564-15572.

(84) Hrubý, A.; Kubelík, I.; Štourač, L. Electrical conductivity and thermoelectric power of heavily doped P-type CdSb. Czechoslovak Journal of Physics 1965, 15, 740-746.

(85) Böttger, P. H. M.; Pomrehn, G. S.; Snyder, G. J.; Finstad, T. G. Doping of p-type ZnSb: Single parabolic band model and impurity band conduction. physica status solidi (a) 2011, 208, 2753-2759.

(86) Irwin, M. D.; Buchholz, D. B.; Hains, A. W.; Chang, R. P. H.; Marks, T. J. pType semiconducting nickel oxide as an efficiency-enhancing anode interfacial layer in polymer bulk-heterojunction solar cells. Proceedings of the National Academy of Sciences 2008, 105, 2783-2787. 
(87) Sato, H.; Minami, T.; Takata, S.; Yamada, T. Transparent conducting p-type NiO thin films prepared by magnetron sputtering. Thin Solid Films 1993, 236, 27-31.

(88) Newman, R.; Chrenko, R. M. Optical Properties of Nickel Oxide. Physical Review 1959, 114, 1507-1513.

(89) Chen, C.; Bobela, D. C.; Yang, Y.; Lu, S.; Zeng, K.; Ge, C.; Yang, B.; Gao, L.; Zhao, Y.; Beard, M. C.; Tang, J. Characterization of basic physical properties of Sb2Se3 and its relevance for photovoltaics. Frontiers of Optoelectronics 2017, 10, $18-30$.

(90) Liu, X.; Chen, J.; Luo, M.; Leng, M.; Xia, Z.; Zhou, Y.; Qin, S.; Xue, D.-J.; Lv, L.; Huang, H.; Niu, D.; Tang, J. Thermal Evaporation and Characterization of Sb 2 Se 3 Thin Film for Substrate Sb2Se3 /CdS Solar Cells. ACS Applied Materials \& Interfaces 2014, 6, 10687-10695.

(91) Shay, J. L.; Tell, B.; Buehler, E.; Wernick, J. H. Band Structure of ZnGeP2 and ZnSiP2 - Ternary Compounds with Pseudodirect Energy Gaps. Physical Review Letters 1973, 30, 983-986.

(92) Bergum, K.; Riise, H. N.; Gorantla, S.; Lindberg, P. F.; Jensen, I. J. T.; Gunnæs, A. E.; Galeckas, A.; Diplas, S.; Svensson, B. G.; Monakhov, E. Improving carrier transport in $\mathrm{Cu} 2 \mathrm{O}$ thin films by rapid thermal annealing. Journal of Physics: Condensed Matter 2018, 30, 075702 .

(93) Tabuchi, N.; Matsumura, H. Control of Carrier Concentration in Thin Cuprous Oxide Cu 2 O Films by Atomic Hydrogen. Japanese Journal of Applied Physics 2002, 41, $5060-5063$.

(94) Heinemann, M.; Eifert, B.; Heiliger, C. Band structure and phase stability of the copper oxides $\mathrm{Cu} 2 \mathrm{O}, \mathrm{CuO}$ and Cu4O3. Physical Review B 2013, 87, 115111. 
(95) Ueda, K.; Hosono, H.; Hamada, N. Energy band structure of LaCuOCh $(\mathrm{Ch}=\mathrm{S}$, Se and Te) calculated by the full-potential linearized augmented plane-wave method. Journal of Physics: Condensed Matter 2004, 16, 5179-5186.

(96) Barati, A.; Klein, A.; Jaegermann, W. Deposition and characterization of highly ptype antimony doped ZnTe thin films. Thin Solid Films 2009, 517, 2149-2152.

(97) Hiramatsu, H.; Ueda, K.; Ohta, H.; Hirano, M.; Kamiya, T.; Hosono, H. Degenerate p-type conductivity in wide-gap LaCuOSSe epitaxial films. Applied Physics Letters 2003, 82, 1048-1050.

(98) Yang, C.; Knei $\beta$, M.; Lorenz, M.; Grundmann, M. Room-temperature synthesized copper iodide thin film as degenerate p-type transparent conductor with a boosted figure of merit. Proceedings of the National Academy of Sciences 2016, 113, 1292912933.

(99) Ahn, D.; Park, S.-H. Cuprous halides semiconductors as a new means for highly efficient light-emitting diodes. Scientific Reports 2016, 6, 20718.

(100) Kawazoe, H.; Yasukawa, M.; Hyodo, H.; Kurita, M.; Yanagi, H.; Hosono, H. P-type electrical conduction in transparent thin films of CuAlO2. Nature 1997, $\underline{389}, 939-942$.

(101) Tate, J.; Ju, H. L.; Moon, J. C.; Zakutayev, A.; Richard, A. P.; Russell, J.; McIntyre, D. H. Physical Review B 2009, 80, 165206.

(102) Kudo, A.; Yanagi, H.; Hosono, H.; Kawazoe, H. SrCu2O2: A p -type conductive oxide with wide band gap. Applied Physics Letters 1998, 73, 220-222.

(103) Ohta, H.; Orita, M.; Hirano, M.; Yagi, I.; Ueda, K.; Hosono, H. Electronic structure and optical properties of SrCu2O2. Journal of Applied Physics 2002, 91, 3074-3078.

(104) Kramida, A.; Ralchenko, Y.; Reader, J.; Team, N. A. NIST Atomic Spectra Database. 2019. 
(105) Kittel, C. Introduction to Solid State Physics, 6th ed.; John Wiley \& Sons, Inc.: New York, 1986.

(106) Stevanović, V.; Lany, S.; Zhang, X.; Zunger, A. Correcting density functional theory for accurate predictions of compound enthalpies of formation: Fitted elemental-phase reference energies. Physical Review B 2012, 85, 115104.

(107) Bismuth sulfide (Bi2S3) energy gaps: Datasheet from Landolt-Börnstein. https: //materials.springer.com/lb/docs/sm_lbs_978-3-540-31360-1_936, Copyright 1998 Springer-Verlag Berlin Heidelberg.

(108) Martinez, L.; Bernechea, M.; De Arquer, F. P. G.; Konstantatos, G. Near IR-sensitive, non-toxic, polymer/nanocrystal solar cells employing Bi 2S 3 as the electron acceptor. Advanced Energy Materials 2011, 1, 1029-1035.

(109) Bismuth selenide (Bi2Se3) energy gaps: Datasheet from Landolt-Börnstein. https: //materials.springer.com/lb/docs/sm_lbs_978-3-540-31360-1_950, Copyright 1998 Springer-Verlag Berlin Heidelberg.

(110) Spataru, C. D.; Léonard, F. Fermi-level pinning, charge transfer, and relaxation of spin-momentum locking at metal contacts to topological insulators. Physical Review B 2014, 90, 085115.

(111) Grüneis, A.; Kresse, G.; Hinuma, Y.; Oba, F. Ionization Potentials of Solids: The Importance of Vertex Corrections. Physical Review Letters 2014, 112, 096401.

(112) V., B.; M.E., L.; S.L., R.; A., Z. In Properties of Advanced SemiconductorMaterials GaN, AlN, InN, M.E., L., S.L., R., M.S., S., Eds.; John Wiley Sons, Inc.: New York, 2001; pp 1-30.

(113) Lim, K.; Schelhas, L. T.; Siah, S. C.; Brandt, R. E.; Zakutayev, A.; Lany, S.; Gorman, B.; Sun, C. J.; Ginley, D.; Buonassisi, T.; Toney, M. F. The effect of sub-oxide 
phases on the transparency of tin-doped gallium oxide. Applied Physics Letters 2016, $\underline{109,} 141909$.

(114) Irmscher, K.; Naumann, M.; Pietsch, M.; Galazka, Z.; Uecker, R.; Schulz, T.; Schewski, R.; Albrecht, M.; Fornari, R. On the nature and temperature dependence of the fundamental band gap of In2O3. physica status solidi (a) 2014, 211, 54-58.

(115) Hohmann, M. V.; Ágoston, P.; Wachau, A.; Bayer, T. J. M.; Brötz, J.; Albe, K.; Klein, A. Orientation dependent ionization potential of In 2 O 3 : a natural source for inhomogeneous barrier formation at electrode interfaces in organic electronics. Journal of Physics: Condensed Matter 2011, 23, 334203.

(116) $\mathrm{MgO}$ energy gap: Datasheet from "PAULING FILE Multinaries Edition - 2012" in SpringerMaterials. https ://materials. springer. com/isp/physical-property/ docs/ppp_88fc7a6adf40f1c09d4946b62a6ad2ba, Copyright 2016 Springer-Verlag Berlin Heidelberg \& Material Phases Data System (MPDS), Switzerland \& National Institute for Materials Science (NIMS), Japan.

(117) Magnesium silicide (Mg2Si) band structure, energy gap: Datasheet from Landolt-Börnstein. https://materials.springer.com/lb/docs/sm_lbs_ 978-3-540-31360-1_100, Copyright 1998 Springer-Verlag Berlin Heidelberg.

(118) Ravich, Y. I.; Efimova, B. A.; Smirnov, I. A. In Semiconducting Lead Chalcogenides; Stil'bans, L. S., Ed.; Springer US: Boston, MA, 1970.

(119) Oman, R. M. Work Function of Lead Sulfide. Journal of Applied Physics 1965, 36, 2091-2092.

(120) Wang, H.; Gibbs, Z. M.; Takagiwa, Y.; Snyder, G. J. Tuning bands of PbSe for better thermoelectric efficiency. Energy Environ. Sci. 2014, $\underline{7}, 804-811$. 
(121) Varley, J. B.; Schleife, A.; Janotti, A.; Van De Walle, C. G. Ambipolar doping in SnO. Applied Physics Letters 2013, 103.

(122) Stevanović, V.; Hartman, K.; Jaramillo, R.; Ramanathan, S.; Buonassisi, T.; Graf, P. Variations of ionization potential and electron affinity as a function of surface orientation: The case of orthorhombic SnS. Applied Physics Letters 2014, 104.

(123) Lany, S. Polymorphism, band-structure, band-lineup, and alloy energetics of the group II oxides and sulfides $\mathrm{MgO}, \mathrm{ZnO}, \mathrm{CdO}, \mathrm{MgS}, \mathrm{ZnS}$, CdS. SPIE Photonics West 2014OPTO: Optoelectronic Devices and Materials. 2014; p 89870K. 